\title{
Analysis of quasi-periodic oscillations of position and brightness of details of the radio sources of the solar active regions based on observations made with the radio heliograph Nobeyama
}

\author{
G.B. Gelfreikh ${ }^{1}$, K. Shibasaki ${ }^{2}$, E.Yu. Nagovitsyna ${ }^{1}$, \\ and Yu.A. Nagovitsyn ${ }^{1}$ \\ ${ }^{1}$ Central astronomical observatory at Pulkovo of RAS, St.-Petersburg, Russia, \\ email: gbg@GG1623.spb.edu \\ ${ }^{2}$ Nobeyama solar radio observatory, Japan
}

\begin{abstract}
As based on analysis of radio maps at the wavelength of $1.76 \mathrm{~cm}$ obtained from observations at the radio heliograph Nobeyama the parameters of oscillation processes in solar active regions were studied. As a technique for data processing wavelet analysis was used. The inherent periodicity in oscillations submits the existence of a resonance structure for some kinds of MHD waves in the plasma of the solar atmosphere.
\end{abstract}

\section{Introduction}

Observations of the quasi-periodic pulsations of the microwave solar radio emission are being made for about 40 years. These were initiated by the group of Prof. Kobrin in Gorkiy (Nizhniy Novgorod) and then developed by the team of the Pulkovo observatory and Siberian Institute of the Solar-terrestrial physics. The most significant quasi-periodic oscillations (QPO) were found to originate from variations of the local radio sources of the solar active regions. Analyzing observations of the QPO as an effective method to study the physics of the solar plasma became especially evident in the course of the last decade due to usage of new large high spatial resolution instruments, the radio heliograph Nobeyama, in particular. Some new results of such research is presented below.

\section{Methods and results}

In observations of the QPO at microwaves we deal mostly with nonstationary processes. These are reasonably to analyze with the help of the wavelet transform

$$
\left[W_{\psi} x\right](a, b)=\int_{-\infty}^{+\infty} x(t) \psi_{a b}^{*} d t=|a|^{-1 / 2} \int_{-\infty}^{+\infty} x(t) \psi^{*}\left(\frac{t-b}{a}\right) d t .
$$

This allows us to study the dynamical spectrum of time series. So, from the wavelet spectrum we win the information both on the amplitude and frequency variations. In this work we have applied the wavelet-approach to research the QPO above different physical structures of the solar atmosphere.

Typical wavelet spectra for large and small sunspots are shown in figure 1. One can see that above sunspot with strong magnetic field dominate oscillations with periods in 


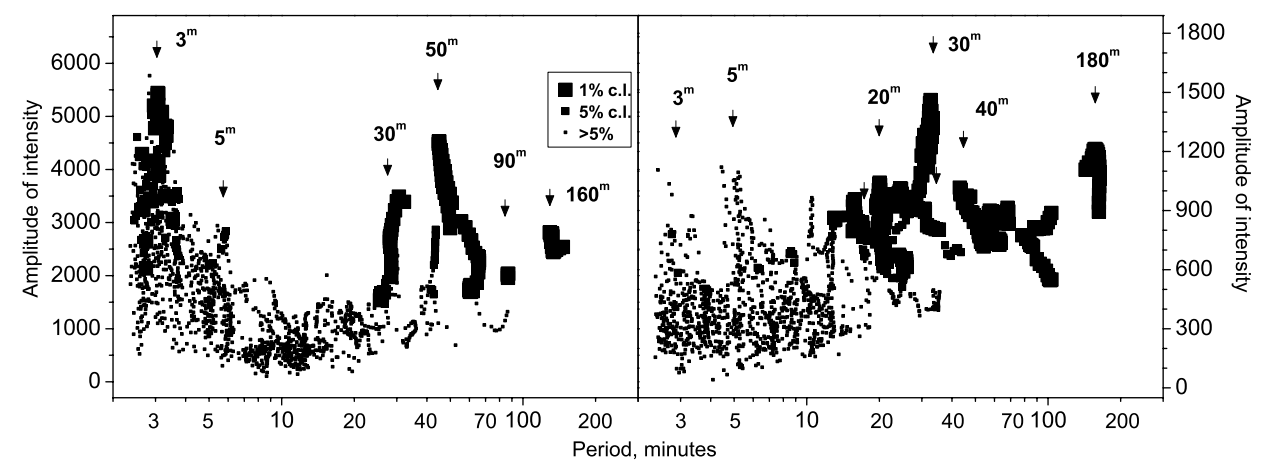

Figure 1. Examples of dependence of amplitude $v s$ period of oscillations for large (left) and small (right) sunspots and confidence levels of QPO

Active structures :

Ranges of periods, minutes :

- sunspots with $B>2500 \mathrm{G} \ldots \ldots 2.5-3,25-30,40$ - 60, 150 - 180

- sunspots with $B<2500 \mathrm{G} \ldots \ldots 15-20,30-35,40-50,160-180$

- bright sources over flocculi .... $10-15,20-30,70-80,100-150$

- polar faculae ......... 3 - 3.5, 5, 10 - 20, 25 - 35, 50 - 70

Table 1. Typical ranges of periods of dominate QPO

the range of $3 \mathrm{~min}$, while the periods of tens and hundreds of minutes are also present. For the case of sunspots with weaker fields $(B<2500 \mathrm{G})$ and for floccular sources periods exceeding 10-15 min were mostly found. Essentially QPO with periods of 40$60 \mathrm{~min}$ are typical (figure 1$)$. In the case of the Nobeyama observations $(\lambda=1.76 \mathrm{~cm})$ absence of the 3-min oscillations above some sunspots may be connected with the weaker thermal gyroresonance emission at the third harmonic of the electron gyrofrequency needed magnetic field $B>2000 \mathrm{G}$ in the hot (coronal) levels of the solar atmosphere.

\section{Conclusions}

One can summarize (see table 1 ) that most oscillations belong to the following ranges of periods $\sim 3, \sim 5, \sim 10,20-25,40-60,80-100,150-180$ minutes. Observed radio oscillations are obviously the result of modulation of physical parameters of the region producing the radio emission. The presence of periodicity in oscillations submits the existence of a resonance structure for some kinds of MHD waves in the plasma of the solar atmosphere. However, the position of the resonator as well as its size may be quite different from those of the microwaves feature of the solar disk under investigation. We may propose 3 main types of the above situation: the resonator coincides with the emitting region; the resonator is outside but close to the radio emitter; the resonator of global solar nature (e.g. 5 min oscillations).

\section{Acknowledgements}

The work was supported by "Astronomy", "Integration" (I0208.1173), "Scientific Schools" (477.2003), Presidium of RAS (4), OFN (16) programs, grants of INTAS (00-0543, 010550), RFBR (02-02-16548, 03-02-17357, 03-02-17528, 04-02-17560). 